\title{
Linx
}

Revue des linguistes de l'université Paris X Nanterre

$74 \mid 2017$

Claudine Normand, une vie dans le langage

\section{Rendre visible des réalités linguistiques}

\author{
Claire A. Forel
}

\section{(2) OpenEdition \\ Journals}

Édition électronique

URL : http://journals.openedition.org/linx/1711

DOI : 10.4000/linx.1711

ISSN : 2118-9692

\section{Éditeur}

Presses universitaires de Paris Nanterre

\section{Édition imprimée}

Date de publication : 31 juillet 2017

Pagination : 67-75

ISSN : 0246-8743

\section{Référence électronique}

Claire A. Forel, «Rendre visible des réalités linguistiques », Linx [En ligne], 74 | 2017, mis en ligne le 15 avril 2018, consulté le 20 avril 2019. URL : http://journals.openedition.org/linx/1711 ; DOI : 10.4000/ linx. 1711 


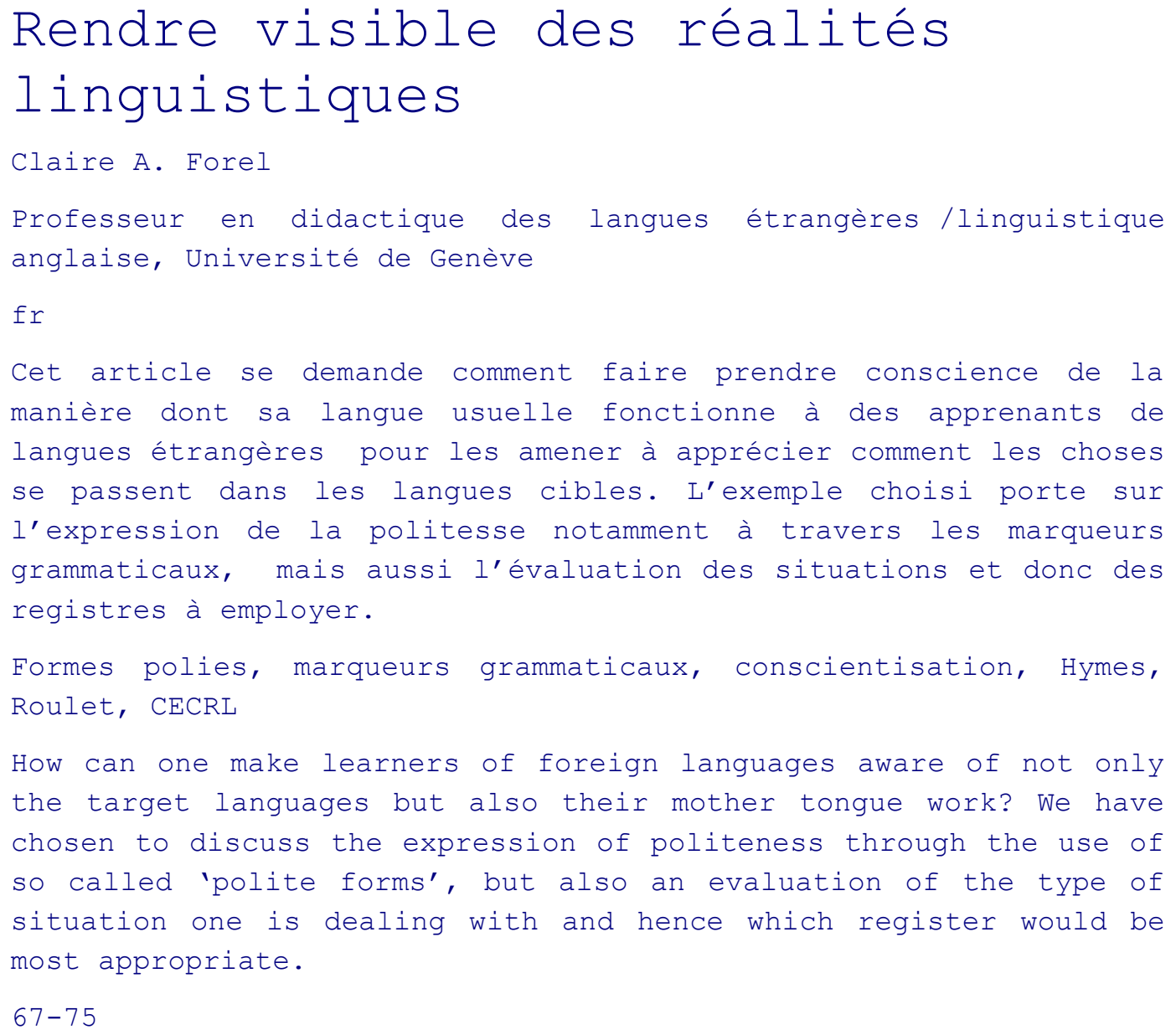

Dans sa Petite Grammaire du Quotidien Claudine Normand se proposait de « rendre visible [...] une réalité singulière [...] qui se manifeste de façon chaque fois particulière et concrète [...] chez celui qui parle sa langue naturellement » (p. 9). Ce que j'aimerais faire ici, c'est montrer comment l'apprentissage d'une langue étrangère vient questionner et, s'il est bien guidé, rendre visible les réseaux d'équivalences et d'oppositions avec lesquels le locuteur natif, dans ce cas francophone, opère parfois sans s'en rendre compte. A terme, cette découverte devrait permettre de comprendre et d'accepter que les langues ont toutes différentes manières de découper l'univers des sons et celui des sens, bref de pouvoir toucher à l'arbitraire du signe.

F. de Saussure faisait de la prise en compte de la diversité des langues le point de départ de la linguistique.

Et le linguiste est dans l'impossibilité d'étudier autre chose au début que la diversité des langues. [...] Par l'étude, l'observation de ces langues, il pourra tirer des traits généraux, il retiendra tout ce qui lui paraît essentiel et universel, pour laisser de côté le particulier et l'accidentel. (E. Constantin, in Cahiers Ferdinand de Saussure (2005 : 89) 
Presque cent ans plus tard, et à propos non pas des scientifiques mais des linguistes en herbe que sont les apprenants de langues étrangères, le Cadre Européen Commun de Référence pour les langues (CECR) du Conseil de l'Europe répond en montrant comment langues étrangères et langue maternelle se combinent chez l'apprenant pour amener à une plus grande conscience linguistique.

\begin{abstract}
On doit cependant apporter une modification importante. L'apprenant d'une deuxième langue (ou langue étrangère) et d'une deuxième culture (ou étrangère) ne perd pas la compétence qu'il a dans sa langue et sa culture maternelles. Et la nouvelle compétence en cours d'acquisition n'est pas non plus totalement indépendante de la précédente. L'apprenant n'acquiert pas deux façons étrangères d'agir et de communiquer. Il devient plurilingue et apprend l'interculturalité. Les compétences linguistiques et culturelles relatives à chaque langue sont modifiées par la connaissance de l'autre et contribuent à la prise de conscience interculturelle, aux habiletés et aux savoir-faire. Elles permettent à l'individu de développer une personnalité plus riche et plus complexe et d'accroître sa capacité à apprendre d'autres langues étrangères et à s'ouvrir à des expériences culturelles nouvelles.
\end{abstract}

(CECR : 40)

Ainsi donc, langue maternelle et langues étrangères sont des systèmes de connaissance qui viennent s'enrichir les uns les autres; le moteur de ce processus est l'appréhension de l'altérité et les prises de conscience multiples qui en découlent; l'ensemble de la démarche favorise de nouvelles compétences. Nous allons en faire la démonstration à travers l'apprentissage par des élèves des établissements secondaires de Genève des personnes verbales en anglais, cette langue venant après l'allemand dans leur cursus.

\title{
1. La forme polie en anglais et ailleurs
}

Le paradigme qu'il convient d'appréhender est le suivant :

\begin{tabular}{|l|l|l|l|}
\hline I & am & do & can \\
\hline You & are & do & can \\
\hline He, she, it & is & does & can \\
\hline We & are & do & can \\
\hline You & are & do & can \\
\hline They & are & do & can \\
\hline
\end{tabular}

Pour ce qui concerne la deuxième personne, you, il n'y a pas de différence formelle entre le singulier et le pluriel et donc ce pronom indique : référence à un/plusieurs interlocuteur/s. Cela ne manque pas d'intriguer les élèves, qui demandent très vite : «Comment on fait pour la forme polie ?». 
Il convient à ce stade de faire une première observation sur le matériau linguistique. Tout comme en anglais, il n'y a en français souvent pas d'inflexion particulière pour une personne verbale : la forme [Jãt] 'chant-' reste identique à l'oral, qu'elle soit précédée des pronoms à la première ou à la deuxième personne du singulier ou à la troisième personne, singulier et pluriel confondus. Par ailleurs, en anglais comme dans beaucoup de langues, avec des déictiques comme you - qui s'applique indifféremment à un ou plusieurs interlocuteurs - il n'y a en général pas de problème pour établir la référence, sauf s'il y a plusieurs interlocuteurs, et ce que l'on dit pourrait s'appliquer à l'un seulement ou à plusieurs d'entre eux. En anglais, pour ce qui concerne la deuxième personne, il y a des cas où cette référence peut être univoque, par exemple quand il y a adjonction d'une copule avec be : You are a nice teacher vs. You are $\emptyset$ nice teachers. Par contre, à l'impératif, en l'absence de pronom, on a aussi qu'une forme Come! qui ne permet de savoir si l'on s'adresse à l'un ou, le cas échéant, plusieurs, interlocuteurs.

Si l'on regarde maintenant les formes dites polies dans d'autres langues connues des élèves, on remarque qu'en français, c'est le pronom 'vous' qui joue ce rôle. L'ambiguïté peut venir de ce que l'on ne sait pas si on s'adresse à une personne à la forme polie, ou à plusieurs d'entre elles, et dans ce cas il n'y a pas de possibilité d'établir si le rapport social est familier ou plus distant. Comme nous l'avons vu pour l'anglais, il y a des formes qui permettent cette distinction : "Vous êtes un bon prof» par opposition à «Tu es un bon prof ». Par contre, dans «Vous êtes de bons profs», il n'est pas possible sur ce seul indice de savoir si le locuteur utilise la forme polie ou non. Il en va de même à l'impératif avec «Venez!». L'allemand connaît lui aussi une forme polie qui 'recycle' un pronom déjà utilisé par ailleurs pour la troisième personne du pluriel :/zi:/. Ce pronom ne se confond pas avec le sie de la troisième du singulier à cause des inflexions différentes du verbe au singulier ou au pluriel ( sie ( $\mathrm{sg}$ ) singt vs. sie ( $\mathrm{pl}$ ) singen). Ainsi, la forme orale [komən zi :] peut se traduire 'Est-ce qu'ils/elles viennent?' (Kommen sie ?) ou 'Est-ce que vous (poli) venez ?' (Kommen Sie ?). Pour cette dernière interprétation on a une ambigüité sur le nombre de personnes (une ou plusieurs) auxquelles on s'adresse, mais il s'agit forcément de la forme polie, parce que pour la forme familière on aurait [komst du] pour le singulier ou [komt i:r] pour le pluriel. Dans Sie sind ein guter Lehrer » c'est forcément la forme polie au singulier. Par contre dans « Sie sind $\emptyset$ gute Lehrer on ne peut faire la différence entre la forme polie au pluriel et la troisième du pluriel, 'Vous êtes de bons profs.'/'Ils sont de bons profs'.

On peut schématiquement présenter sous forme de tableau ce que nous venons d'exposer.

\begin{tabular}{|l|l|l|l|}
\hline & français & anglais & allemand \\
\hline $2^{\text {ème }} \mathrm{sg} / \mathrm{pl}$ & tu/vous & (neutralisé) you & $\mathrm{du} / \mathrm{ihr}$ \\
\hline Forme polie & vous & --- & $\mathrm{Sie}$ \\
\hline Forme polie s'oppose à & vous $: 2^{\text {ème }}$ pluriel & ---- & $\mathrm{sie}: 3^{\text {ème }}$ pluriel \\
\hline
\end{tabular}




\begin{tabular}{|l|l|l|l|}
\hline $\begin{array}{l}\text { Ambiguité forme polie } \\
\text { avec... }\end{array}$ & $\begin{array}{l}2^{\text {ème }} \\
\text { 'Vour } \\
\text { souvent?' }\end{array}$ & $\begin{array}{l}3^{\text {ème }} \text { pluriel } \\
\text { Kommen S/sie oft ? }\end{array}$ \\
\hline Univocité formelle & $\begin{array}{l}\text { 'Vous êtes un bon } \\
\text { prof.' }\end{array}$ & $\begin{array}{l}\text { You're a good } \\
\text { teacher. }\end{array}$ & $\begin{array}{l}\text { Sie sind ein guter } \\
\text { Lehrer. }\end{array}$ \\
\hline
\end{tabular}

A la remarque qu'il n'y a pas de «forme polie » en anglais, on répondra en amenant les élèves à s'interroger sur ces formes dans les autres langues connues de tous les élèves : le français langue de scolarisation et l'allemand dont l'étude est obligatoire en Suisse romande. Soit le maître fournit des données, soit il en fait produire. Une discussion peut alors d'engager. Elle peut montrer que ladite forme polie est comprise sans problème la plupart du temps grâce au contexte, mais alors il faut s'interroger sur les indices que l'on utilise pour décoder celui-ci. Lorsque le contexte est peu clair, il peut y avoir des ambiguïtés dues à la forme. Le but de cette démarche est de faire prendre conscience de la différence dans une interaction entre les informations fournies par le code et celles qui viennent du contexte ou d'autres connaissances. La discussion permettra peut-être aussi de faire émerger ce nouvel usage du 'tu' français, qu'on voit apparaître lorsqu'un locuteur qui normalement vouvoie son interlocuteur passe au tutoiement, par exemple pour mieux l'impliquer dans son récit, réduisant ipso facto la distance qui les sépare.

\section{Politesse en l'absence de formes polies en anglais}

L'usage de formes polies ne suffit pas pour qu'il y ait politesse. La question de la politesse est difficile, il suffit de lire V. P. Brown and S. Levinson 1987 pour bien s'en convaincre. Par contre, la notion de 'pouvoir et de solidarité' à travers l'usage des formes en 'tu' et 'vous' que R. Brown et A. Gilman ont exposée dans un article devenu un classique, nous permettra de montrer comment on peut parler du sujet dans une classe. On peut la résumer en disant que la forme familière montre une certaine solidarité, la forme polie exprime soit une absence de solidarité quand elle est réciproque, soit une relation de pouvoir quand elle n'est pas réciproque : le supérieur marquant son statut en tutoyant son inférieur qui le vouvoie. C'est bien ce qui se passe entre un enseignant et son élève du moins jusque dans le secondaire inférieur.

Voyons comment l'anglais s'y prend pour marquer les rapports de solidarité ou d'absence de celle-ci, malgré l'absence de 'forme polie'. L'exemple ci-dessous repris dans d'un manuel un peu ancien, n'a néanmoins rien perdu de son actualité. 
2 @isten. Learn the new words from a dictionary or from your teacher. Listen again and practise.
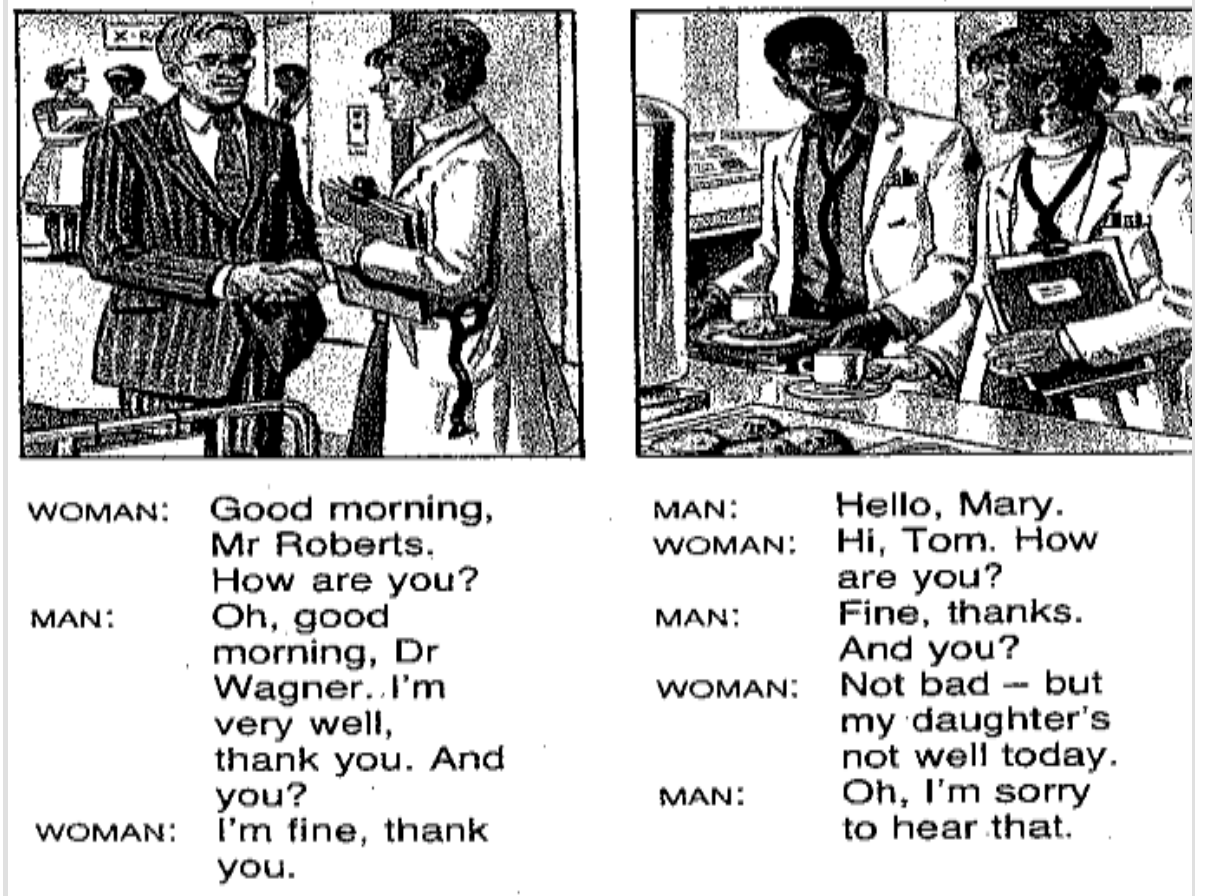
Mr Roberts. How are you? MAN: Oh, good morning, Dr Wagner..I'm very well, thank you. And you?

WOMAN: l'm fine, thank you.

Les élèves sont tout d'abord invités à identifier les situations qui leur permettront d'opposer une forme plus formelle à l'unique formule familière : 


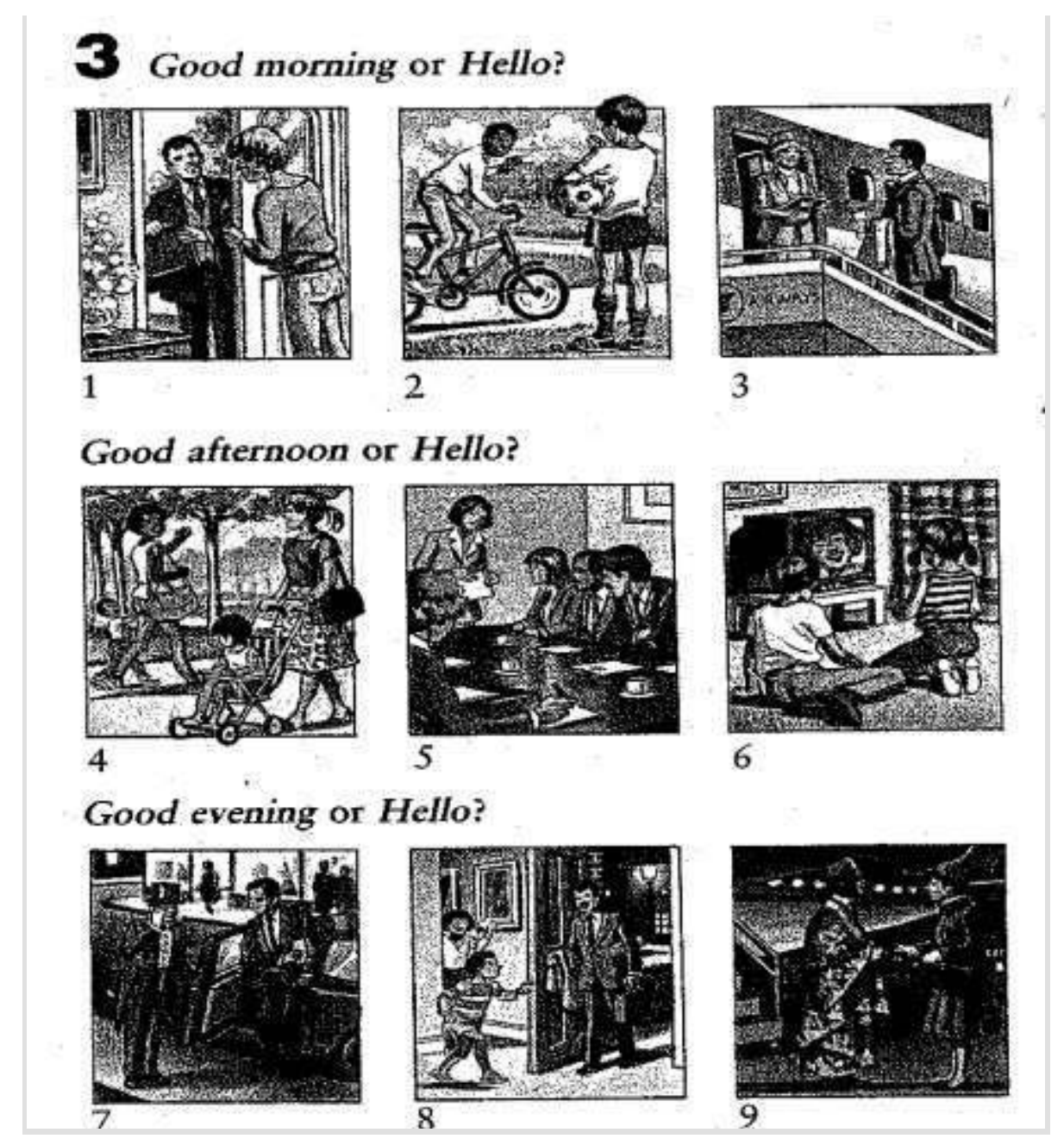

Si l'on se concentre sur le dialogue de départ on remarque rapidement des différences de quatre ordres. Les premières ont trait aux formules de salutation avec le Good morning qui s'oppose aux Hello, comme dans les petites vignettes ci-dessus. Il y a ensuite l'usage très anglo-saxon des titres ou, à l'inverse, des prénoms. Jusque là rien que d'assez évident, même pour ce qui touche des élèves adolescents. Ce sont des codes dont ils sont assez conscients. Peut-être n'en va-t-il pas tout à fait de même pour ce qui touche au sujet de la conversation, dont la référence à sa famille rend l'échange avec Tom singulièrement plus intime, par contraste avec la neutralité parfaite de l'autre échange. Enfin, la forme plus ou moins développée des phrases est aussi remarquable : si Mary Wagner répondait à la question de $\mathrm{Mr}$ Roberts How are you? Fine, thanks and you ? cela indiquerait un autre type de rapport entre eux. On peut évoquer deux raisons : l'une tient à la forme familière de remerciement thanks au lieu de thank you, et l'autre au renvoi de la question and you qui supposerait un rapport de réciprocité ; l'une et l'autre ne sont pas de mise dans un tel rapport de hiérarchie. En résumé, en l'absence d'une forme polie liée au verbe, c'est probablement l'ensemble de ces facteurs qui caractérisent nos deux échanges comme relevant de la solidarité ou de son absence. Dans une langue comme le français ou l'allemand, la présence de la forme polie ou, au contraire, de la forme familière est le trait le plus saillant pour déterminer le type de rapport social entre les participants. Ce qui n'empêche pas que les autres éléments que nous avons vus mis en œuvre en anglais soient aussi présents, de manière propre à chaque langue. 


\section{Langue de scolarisation et langues étrangères}

Eddy Roulet, dans Langue maternelle et langues secondes, vers une pédagogie intégrée parle de «procédure de découverte rétrospective du fonctionnement de la langue maternelle et de la langue seconde à partir de données de la langue seconde qui est très fructueuse » (Roulet, 1980 : 115). Cela convient bien à ce que l'on peut faire dans une situation comme celle que nous venons de voir à propos des formes polies dans la langue cible puis en langue maternelle et dans une autre langue étrangère. Toutefois, d'une manière générale, dans le reste de son livre, il préconise pour les langues secondes une réactivation des procédures heuristiques utilisées préalablement dans l'étude de la langue maternelle. Comment expliquer que, dans le cas qui nous intéresse, il faille passer par la langue étrangère pour mieux faire comprendre ce qui se passe dans la langue de scolarisation? Hymes parle de «the internalization of attitudes towards a language and its uses is particularly important» (Hymes, 1972: 278). Il explique que «The acquisition of such competency is of course fed by social experience, needs, and motives, and issues in action that is itself a renewed source of motives, needs, experience" (Hymes, 1972: 278).

La connaissance de la langue maternelle et de ses règles de fonctionnement est le plus souvent implicite. Ce n'est pas à l'école, quoi qu'on en dise, qu'on est le plus massivement mis en contact avec les règles de fonctionnement de la société, mis à part les règles locales nécessaires au fonctionnement de ce type d'établissement. C'est en se frottant au monde qu'on en prend conscience mais cela ne fait souvent pas l'objet d'une explicitation. Alors que pour la première langue étrangère, c'est en quelque sorte l'inverse : on a conscience que les choses s'y passent différemment, avec plus ou moins de profondeur de champ, et on doit apprendre à la fois les éléments linguistiques et les éléments sociolinguistiques, voire les éléments sociaux tout court, qui définissent la nouvelle situation. Le passage de la langue de tous les jours à la langue étrangère, lorsqu'il est conscientisé, permet l'émergence concrète de l'interculturalité. Il reste toutefois un écueil à éviter. L'autre manière de faire les choses' présente en langue étrangère pourrait apparaître comme une manière regrettable de procéder, une déviance par rapport à la manière 'naturelle' de procéder qui a cours dans le groupe auquel on appartient. Pour qu'il y ait un véritable plurilinguisme - et une pluriculturalité qui en découlerait -, il faut chercher un troisième point de repère, comme une autre langue étudiée dans le cursus scolaire, ou alors on peut entamer une discussion sur leurs langues maternelles avec des élèves allophones de la classe par exemple. Cela permettrait de passer d'une posture qui constate simplement que les locuteurs de la langue cible font les choses différemment de nous à la compréhension et, espérons-le, l'acceptation que chaque langue a sa manière de faire les choses. C'est bien ce que j'ai tenté de montrer avec you, 'vous' et Sie/euch ainsi qu'avec un exemple de la manière dont l'anglais s'y prenait pour montrer le degré de solidarité entre les interlocuteurs en présence.

Remarquons enfin que si la question de l'expression de la politesse est pertinente pour tous les apprenants en âge de comprendre cette notion (en excluant donc les jeunes enfants), il 
n'est pas évident que les mises en situation qui permettent de l'appréhender soient parlantes pour tous les publics. La leçon du manuel que nous avons examinée n'est pas forcément très significative pour des adolescents. La prise de conscience que nous revendiquons doit s'adapter au niveau de l'expérience réelle des élèves, avec leurs motivations et leurs besoins, et bien définir la manière dont les choses se passent dans leur groupe social d'appartenance et comment elles se passeraient dans la langue étrangère, que ce soit dans un groupe identique ou dans un groupe différent.

\section{Conclusion}

Cette particularité de la morphologie de l'anglais de ne pas avoir de forme verbale dite 'polie' ouvre la fenêtre sur le monde de la langue en action et permet une première prise de conscience sur le monde social et son organisation ainsi que sur la manière dont la langue y contribue. Dans la description de la connaissance d'une langue étrangère qu'est le Cadre Européen, notre petite démonstration relève de la «Compétences sociolinguistiques » qui concerne « la connaissance et les habiletés pour faire fonctionner la langue dans sa dimension sociale » (CECR : 93) : dans notre discussion, nous avons bien touché aux «marqueurs des relations sociales» (5.2.2.1), aux «règles de politesse» (5.2.2.2) et aux «différences de registre » (5.2.2.4). Si la capacité à « jouer efficacement les médiateurs entre des locuteurs de la langue cible et celle de sa communauté d'origine en tenant compte des différences socioculturelles et sociolinguistiques » se situe tout en haut (C2) d'une échelle étalonnant cette compétence, le niveau élémentaire (A1) stipule: «Peut établir un contact social de base en utilisant les formes de politesse les plus élémentaires ; accueil et prise de congé, présentations et dire 'merci', 's'il vous plaît', 'excusez-moi', etc. ». C'est bien ce que propose de faire la leçon que nous avons utilisée dès la sixième heure d'enseignement d'un cours de débutants permet de mettre en place dès le début de l'apprentissage. Ainsi on peut comme le faisait Claudine Normand à propos d'autres exemples, « rendre visible [...] une réalité singulière $[\ldots]$ qui se manifeste de façon chaque fois particulière et concrète $[\ldots]$ chez celui qui parle sa langue naturellement » et utilise la forme polie, pour mieux lui permettre de comprendre ce qui se passe dans d'autres idiomes.

Brown, R \& Gilman, A., 1960, “The pronouns of power and solidarity”, in Sebeok, T. A. (ed.), 1960, Style in Language, Cambridge: MIT press, pp. 253-76.

Cahiers Ferdinand de Saussure. Volume 58 (2005). Edition des notes d'Emile Constantin du Troisième Cours de Linguistique Générale (1910-1911). Librairie Droz.

Conseil de l'Europe, 2001, Cadre européen commun de référence pour les langues: apprendre, enseigner, évaluer, Strasbourg, Conseil de l'Europe / Paris : Didier.

Forel, F., Gerber, B., Rossi F., 2013, «Plaidoyer pour un décloisonnement des apprentissages linguistiques », Rassegna Italiana di Linguistica Applicata 2-3 
HyMES, D., 1971, «On Communicative competence », reproduit dans Pride and Holmes 1972 : Sociolinguistics, Harmondsworth, Penguin, p. 269-293.

NoRMAND, C., 2010, Petite grammaire du quotidien, Paris, Hermann.

RouLET, E. 1980, Langue maternelle et langues secondes: vers une pédagogie intégrée, Paris, Hatier 